\title{
Proposta de interdisciplinaridade entre matemática e física resultando na aprendizagem contextualizada
}

\author{
Feroni, R. C. ${ }^{1,2}$; Andreão, W. L. ${ }^{1}$; Galvão, E. S. ${ }^{1}$ \\ ${ }^{1}$ Programa de Pós-Graduação em Engenharia Ambiental, Universidade Federal do Espírito Santo, Vitória, ES, Brasil. \\ ${ }^{2}$ Departamento Regional da Bahia, Centro Integrado de Manufatura e Tecnologia, Salvador, BA, Brasil. \\ *e-mail: ritaferoni@gmail.com
}

\begin{abstract}
Resumo
A interdisciplinaridade permite que as disciplinas sejam estudadas relacionando-se umas com as outras, mostrando que os conteúdos ministrados estão interligados. Por exemplo, cada indivíduo está cercado de informações que necessitam de interpretação envolvendo o sentido visual e o senso crítico, incluindo aqui os fênomenos físicos. Assim, os conceitos de física, podem ser representados através de uma modelagem matemática e lidos na forma de equações, funções matemáticas e gráficos, ou seja, a física pode utilizar a linguagem simbólica da matemática. Para mostrar a interdisciplinaridade que existe em conteúdos desenvolvidos no ensino da matemática e da física, tanto a nível de ensino médio quanto em cursos de graduação, uma análise preliminar foi realizada no presente trabalho para fundamentar uma investigação no que se refere ao ensino dessas subáreas das ciências exatas. Dessa forma, na opinião dos autores deste trabalho, a interdisciplinaridade entre a matemática e a física promove um ganho no processo de ensino-aprendizagem.
\end{abstract}

\begin{abstract}
Interdisciplinary studies allow to link subjects in differents fields of sciences. All the time we are stimulated by information data that require interpretation using visual sense and critical thinking, including physical phenomena. Therefore, the concepts of physics may be represented by a mathematical model and interpreted in the form of equations, mathematical functions and graphs, namely, the physical may use the symbolic language of mathematics. Thus, to show the interdisciplinary in content developed in the teaching of mathematics and physics, both in the high school and undergraduate courses, a proposal of research was carried out in this paper to investigate the teaching of these sub areas of exact sciences. Thus, with the proposed paper, is verified what are the possible contributions of the use of interdisciplinary between mathematics and physics in the learning process, since exploring the application of the proposed content can to improve student attention to what it is exposed.
\end{abstract}

Keywords (Palavras chaves): interdisciplinaridade, aprendizagem, contextualização, física, matemática.

\section{Introdução}

Corriqueiramente um professor observa que 0 aluno compreende um conceito da física mas apresenta dificuldades nos cálculos, representações ou interpretação necessárias para desenvolver o problema proposto. Apesar de a matemática e a física serem disciplinas estudadas separadamente, e ministradas muitas vezes por professores com diferentes formações acadêmicas, elas possuem aplicações conjuntas. Assim, um projeto de interdisciplinaridade entre estes conteúdos contribuiria para o aprendizado do aluno.

As primeiras abordagens sobre interdisciplinaridade surgiram no Brasil a partir da Lei de Diretrizes e Bases 
(LDB) № 5.692/71 [1], e vem sendo exploradas no contexto da aprendizagem. Cada disciplina tem suas especificidades mas ao mesmo tempo se complementam [2]. A postura e a atitude interdisciplinar visam garantir uma atuação mediadora do professor para facilitar a aprendizagem [3].

A interdisciplinaridade pode proporcionar um trabalho dinâmico e facilitador da aprendizagem, pois trabalha com componentes curriculares interligados entre si, além de possibilitar uma maior motivação dos alunos envolvidos [4]. Em se tratando de matemática e física, a interdisciplinaridade torna-se ainda mais viável, pelo fato da física se utilizar de recursos matemáticos para qualificar e quantificar fenômenos cotidianos.

Neste contexto, o objetivo desta pesquisa é o de mostrar a interdisciplinaridade entre os conteúdos de matemática e física, para construir um conhecimento integrado, aplicado a realidade e, consequentemente mais sólido e contextualizado, ajudando na aprendizagem.

\section{Desenvolvimento}

A física se utiliza da linguagem simbólica da matemática, que dispõe de um conjunto de ferramentas e permite a aquisição de competências, como, por exemplo, o raciocínio lógico, as técnicas de resolução de problemas, e a capacidade de pensar em termos abstratos inerentes ao ensino da física.

Existe uma ampla aplicação de conteúdos correlacionados entre as duas disciplinas. Nesse contexto, a nível de exemplificação pode-se destacar:

(a) A representação, construção e interpretação de funções em conjunto com a parte gráfica, constitui um dos recursos mais eficientes no estudo de fenômenos físicos. A determinação dos pontos em que uma função atinge máximos ou mínimos, é importante do ponto de vista da obtenção de rendimento máximo em qualquer máquina ou análise de movimento harmônico simples. (b) Outra ferramenta matemática essencial no estudo da física é a trigonometria. Pode-se destacar, por exemplo, o estudo do movimento de um projétil lançado horizontalmente ou obliquamente, quando se pretende determinar a direção da velocidade do projétil ao atingir o solo. A aplicação também é encontrada no estudo da reflexão e refração com estudo de ângulos, assim como de movimento circular, ao se identificar o valor da velocidade angular média.

(c) Na geometria, as formas geométricas que aparecem com maior frequência no estudo da física são: a parábola, que pode identificar a trajetória de um projétil, e a circunferência, que aparece no estudo da trajetória do movimento circular e uniforme.

(d) A noção e operações envolvendo vetores é uma das ferramentas essenciais no estudo da física.

(e) A função logarítmica de base 10 é também uma função importante que surge no estudo do som. Temse, como exemplo, o decibel $(\mathrm{dB})$ que é uma unidade logarítmica muito usada no estudo do som e em telecomunicações.

(f) A estatística é um campo importante da matemática, que permite a descrição, análise e previsão de fenômenos aleatórios, e é usada bastante em análise de experimentos físicos.

\subsection{Exemplo de aplicação}

Como visto anteriormente, existem muitos conteúdos que podem ser ministrados interdisciplinarmente entre os professores de matemática e física.

Um exemplo de aplicação são as funções matemáticas polinomiais de $1^{\circ}$ e $2^{\circ}$ grau, que relacionam-se com as funções cinemáticas obtidas dos movimentos uniforme e uniformemente variado, respectivamente. A função estudada na matemática, como a função (1), função polinomial do $1^{\circ} \mathrm{grau}$, é equivalente a função física que representa a posição de um móvel no movimento 
uniforme em função do tempo, conforme se observa em (2).

$$
\begin{aligned}
& f(x)=a x+b \\
& s(t)=s_{0}+v t
\end{aligned}
$$

Assim, para exemplificar, pode-se considerar a Figura 1 , referente ao gráfico da função, $f(x)=2 x+1$. O gráfico refere-se a uma função polinomial do $1^{\circ} \mathrm{grau}$, ou seja, do tipo $f(x)=a x+b$, sendo uma reta crescente.

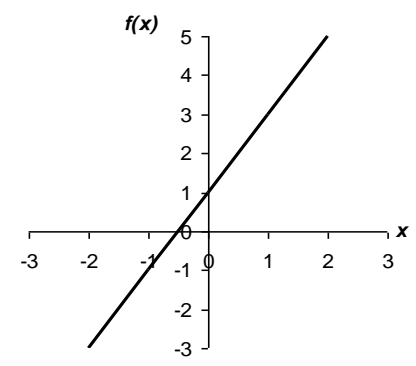

Figura 1: Gráfico de função puramente matemática

Pode-se contextualizar o problema anterior, puramente matemático, com a seguinte situação física: Um móvel desloca-se sobre uma reta de acordo com o gráfico ilustrado na Figura 2.

Em uma análise direta, é possível identificar alguns pontos importantes como em $t_{0}=0 \mathrm{~s}$, o móvel ocupa a posição $s(0)=1 m$ e em $t_{1}=1 s$, o móvel se deslocou para a posição $s(1)=3 m$ e assim por diante.

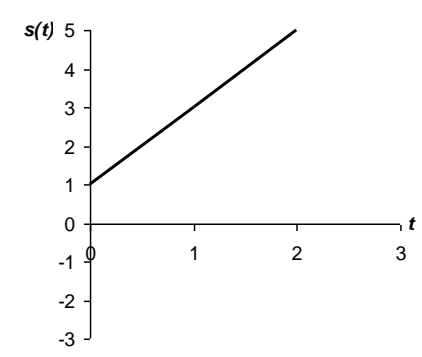

Figura 2: Gráfico de função com interpretação física.

A equação de movimento do móvel é dada pela Equação (2), ou escrita para o problema proposto, $s(t)=1+2 t$.
As associações matemáticas não existem somente relacionadas a análise gráfica, pode-se considerar que existe uma equivalência entre o coeficiente linear, $b=1$, que fisicamente representa a posição de origem do movimento, ou seja, $s_{0}=1 \mathrm{~m}$. O coeficiente angular, $a=2$, é um valor relacionado a velocidade do móvel igual a $v=2 \mathrm{~m} / \mathrm{s}$. O movimento do móvel é progressivo, pois a sua velocidade $v$ é positiva. Dessa forma, tem-se as correlações matemáticas e físicas relacionadas a função.

\section{Conclusões}

Este estudo surgiu com a proposta de mostrar a interdisciplinaridade de conteúdos relacionados ao estudo da matemática e física. A partir do exposto, é possível verificar a possibilidade das disciplinas de matemática e física serem ministradas em conjunto com um plano de aula estruturado pelo professor. $\mathrm{Na}$ opinião dos autores deste trabalho, essa é uma alternativa viável para melhorar o desempenho dos alunos nas disciplinas de ciências e, de modo particular, em ciência física.

\section{Agradecimentos}

Os autores agradecem a CAPES pelo apoio financeiro, e aos professores Marcos T. D. Orlando e Carlos A. C. Passos pelas contribuições.

\section{Referências}

[1] BRASIL. Senado Federal. Lei de Diretrizes e Bases da Educação Nacional: no 5692/71. Brasília, 1971. Disponível em <www.planalto.gov.br/ccivil_03/leis/L5692.htm>. Acesso em: 09 de maio de $\overline{2016 .}$

[2] PACHECO, R. C. et al. RBPG, Brasília, v.7, p.136 159, 2010.

[3] NOGUEIRA, N. B. Pedagogia dos Projetos: Uma jornada interdisciplinar rumo ao desenvolvimento das múltiplas inteligências. São Paulo: Érica, 2007.

[4] Rosa, C. P. et al. Matemática e física: juntas pela função afim. III Escola de Inverno de Educação Matemática e $1^{\circ}$ encontro Nacional PIBID- Matemática. Santa Maria, 2012. Disponível em < http://w3.ufsm.br/ceem/eiemat/Anais/arquivos/RE/RE_ 
Rosa_Carine_Pedroso.pdf $>$. Acesso em: 05 de maio de 2016. 